phytol and phytanic acid.(Progress in Pediatric Neurology, Chicago, PNB Publ, 1991, p 480).

\title{
CEREBELLAR ATAXIA AND MULTIPLE SCLEROSIS
}

Clinical manifestations and MRI findings in four Japanese children with multiple sclerosis are reported from the Department of Pediatrics, Sapporo Medical University, Japan. Three presented with gait ataxia and one developed cerebellar intention tremor within 2 months of an onset with weakness of the right arm and speech impairment. The age of onset of symptoms was at 7-12 years. All had optic neuritis. MRI showed multiple white matter lesions and demyelinating plaques in cerebral hemispheres, cerebellum and brain stem. CT abnormalities were indefinite or absent.(Wakai $S$ et al. Childhood multiple sclerosis: MR images and clinical variations in four Japanese cases. Brain Dev 1994; $16: 52-56$ ). (Respond: Dr S Wakai, Dept of Pediatrics, School of Medicine, Sapporo Medical University, South 1 West 16, Chuo-ku, Sapporo 060, Japan).

COMMENT. MRI was more sensitive than CT in diagnosis of demyelination in these patients. One of the 4 children had presented with Devic disease and 2 years later developed chronic inflammatory demyelinating polyradiculoneuropathy. A survey of 55 pediatric patients with MS in Japan by Prof Y Fukuyama and associates (1991) had found peripheral nerve involvement in $10(17 \%)$.

\section{TOXIC DISORDERS}

\section{COCAINE EXPOSURE IN CHILDHOOD: NEUROLOGIC SIGNS}

Neurologic manifestations of cocaine exposure in $19(46 \%)$ of 41 children, ages 2 months to 18 years, found to have cocaine-positive urine screening tests during a 1-year period (Jan - Dec 1990), are reported from the Children's National Medical Center, Washington, DC. Seizures were the most common symptom of neurotoxicity, occurring in 7 children in the age range 1-8 years. Obtundation occurred in 6 (ages 5 mos-18 yrs); delirium in 4 (ages 16-19 yrs); dizziness 1 and drooling 2 (ages 16-18 yrs). Seizure patterns were focal with secondary generalization in 3 , generalized in 4 , and were associated with fever in 2. Passive intoxication in a closet where "crack" was smoked was the most likely cause of exposure in young children. An additional 14 adolescent patients with positive urine screens had neurologic complications of head injuries, and cocaine-related symptoms could not be evaluated. (Mott $\mathrm{SH}$ et al. Neurologic manifestations of cocaine exposure in childhood. Pediatrics April 1994;93:557-560). (Reprints: Dr Stephen H Mott, Neurology Dept, Children's National Medical Center, 111 Michigan Ave, NW, Washington, DC 20010).

COMMENT. Seizures were the commonest manifestation of cocaine exposure and neurotoxicity in young children. Adolescents suffered from alterations in mental status. Urine screen for possible cocaine exposure is recommended in children with first-time seizures, afebrile or febrile.

A study of the relationship between maternal cocaine dependency and child maltreatment at Yale University School of Medicine showed that 47 infants cocaine-exposed in utero were at 
increased risk of maltreatment (physical abuse, sexual abuse, or neglect), and placement either in foster care or with a substitute caretaker during the first 24 months of life. (Wasserman DR, Leventhal JM. Maltreatment of children born to cocaine-dependent mothers. AIDC Dec 1993; 147:1324-1328).

The nonspecific nature of symptoms of cocaine poisoning in infants is stressed in a case-report from Duke University School of Medicine. A 5-month-old male presented with lethargy, unresponsiveness, vomiting, and diarrhea. A urine screen was positive for benzoylecgonine, a cocaine metabolite. (Edell DS, McSwain M. Cocaine poisoning: an easily missed diagnosis in an infant. Clin Pediatr Dec 1993; 32:746-747).

An evaluation of the 3-year behavioral and developmental outcome of 93 children exposed prenatally to cocaine and other drugs taken by the mother during pregnancy is reported from the National Association for Perinatal Addiction Research and Education, Chicago, IL. Cocaine exposure predicted poor verbal reasoning on the Stanford Binet. Marijuana exposure predicted poor abstract/visual reasoning. (Griffith DR, Azuma SD, Chasnoff IJ. Three-year outcome of children exposed prenatally to drugs. I Am Acad Child Adolesc Psychiatry Jan $1994 ; 33: 20-27)$. Women who use cocaine during pregnancy also use more tobacco, alcohol, and marijuana than non-cocaine users. When these differences between exposure groups were controlled, there was no significant effect of prenatal cocaine use on infant growth and morphology. (Richardson GA, Day NL. Detrimental effects of prenatal cocaine exposure: Illusion or reality? LAm Acad Child Adolesc

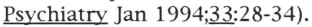

\section{SEIZURE DISORDERS}

\section{VIDEO GAME-RELATED SEIZURES}

The manifestations of video game-related seizures (VGRS) in 10 patients aged 1-22 years examined and 25 case-reports reviewed are reported from the University of Washington School of Medicine, Seattle, WA. Prior susceptibility to epilepsy was evident in 8 patients with infrequent nonfebrile seizures, 4 with febrile seizures, and 2 with a positive family history, a total of $14(40 \%)$. VGRS patterns were generalized tonic-clonic in $22(63 \%)$, simple partial in 6 (19\%), complex partial in 4 (11\%), and absence in 2 (6\%). Electroencephalographic epileptic patterns occurred in 11 of 21 patients $(52 \%)$ and photoparoxysmal responses in 17 of $32((53 \%)$. Treatment was successful in 11 of $15(73 \%)$ who only abstained from video game playing, in 3 of 6 who continued playing VGs and received anticonvulsant drugs, and 7 of 12 who abstained from VGs and also received AEDs. (Graf WD et al. Video game-related seizures: a report on 10 patients and a review of the literature. Pediatrics April 1994;93:551-556). (Reprints: Dr William D Graf, Division of Congenital Defects, Children's Hospital and Medical Center, 4800 Sand Point Way NE, Mail Stop CH-47, Seattle, WA 98105).

COMMENT. Abstinence from playing or watching video games is the treatment of choice for patients with VGR seizures. Anticonvulsant 